\title{
A Summary of the Research on the Judicial Application of Artificial Intelligence
}

\author{
Gulimila Aini \\ Beijing Normal University Law School, Beijing, China \\ Email:na.jiang@bnu.edu.cn
}

How to cite this paper: Aini, G. (2020). A Summary of the Research on the Judicial Application of Artificial Intelligence. Chinese Studies, 9, 14-28.

https://doi.org/10.4236/chnstd.2020.91002

Received: January 21, 2020

Accepted: February 24, 2020

Published: February 27, 2020

Copyright $\odot 2020$ by author(s) and Scientific Research Publishing Inc. This work is licensed under the Creative Commons Attribution International License (CC BY 4.0).

http://creativecommons.org/licenses/by/4.0/

\section{(c) (i) Open Access}

\begin{abstract}
The use of artificial intelligence technology in the field of judicial trials is becoming more widespread. Intelligent software includes functional modules, such as guidance, prompting, and deviation warning, which can assist trials judges in sentencing, case push, and automatic document generation throughout the litigation process, thereby helping to save litigation costs and improve trial efficiency. However, in the application process, intelligent auxiliary software has displayed essential, prerequisite, procedural, and result defects and thus poses ethical risks and challenges. Therefore, we should clarify such technology's auxiliary trial status, determine the scope of specific applications, and formulate ethical norms for judicial artificial intelligence. Additionally, differential trials and post hoc monitoring should be used to standardize the application of such technology.
\end{abstract}

\section{Keywords}

Artificial Intelligence, Judicial Decision, Decision Support

\section{Introduction}

The application of the breakthrough development of intelligent technology has occurred in the judicial field. On January 29, 2016, at a plenary meeting of the Leading Group of the Supreme People's Court's informationization research, President Zhou Qiang first proposed the concept of a smart court. He points out that the smart court is a people's court with the form of organization, construction, operation, and management, which supported by artificial intelligence, paid attention to the principles of "People's Judicatory" and "Judicial Justice", adhere to principle of judicatory, the structural reform, also the technological change integration, support judicial trial, litigation service, and judicial management in a highly computerized way, and implement the online processing of 
the whole business, publicly process according to law and omni-directional smart service (Zhao, 2017). The smart court is listed as an important informationization goal. On January 9, 2018, the Ministry of Science and Technology released the National Key R \& D Plan (Judicial Thematic Task), and the first tranche of 450 million yuan was invested to study the scientific and technological issues facing courts, procuratorates and judicial departments. In this context, courts at all levels have also engaged in a wave of smart court construction. Previously developed artificial intelligence products have entered the judicial field, and a platform for the integration of technical staff and senior judges has been established.

Intelligent software can assist judges to retrieve legal information, guide them to collect and review evidence comprehensively according to the evidence standard and even predict judgment results and generate judgment documents. The application of artificial intelligence in the judicial field responds to a certain extent to the expectations of judicial personnel and the public for intelligent justice. The application of judicial artificial intelligence products can improve the efficiency of trials, ensure the quality of cases, and unify the scale of adjudication. However, the unlimited application of may subvert the inherent attributes of justice and the classic paradigm of justice. In view of these issues, this paper attempts to return to the nature of judicial rule to examine the current status and problems of the judicial application of artificial intelligence. By investigating such technology's development prospect from the perspective of legal subject theory, legal ethics and legal logic, this paper seeks to establish a foundation for the future development of artificial intelligence in the judicial field.

\section{Application Status of Artificial Intelligence in the Judicial Field}

\subsection{Overview of Research and Application of Judicial Artificial Intelligence}

\subsubsection{Theoretical Research Level}

The earliest contribution is by Professor Qian Xuesen, who proposed the general idea of combining artificial intelligence and the law in 1981. A research project entitled "Research on the comprehensive balance of sentencing and computerassisted sentencing expert system", chaired by Zhu Huarong and Xiao Kaiquan, was held in 1986. In 1986, Professor Su Huiyu et al. co-authored "Sentencing and the Computer" and published other theoretical research results, which established the foundation for the early emergence and development of artificial intelligence judicial research and made other important contributions. The most representative paper is by Professor Zhang Baosheng. From the perspective of legal theory, the theoretical and practical value of the research and development of artificial intelligence for the legal system is analysed, and the jurisprudence foundation of artificial intelligence applied in the field of jurisprudence and the research space for jurisprudence are discussed (Zhang, 2001). 
Using the keywords of "artificial intelligence" and "judiciary" to perform a quantitative analysis of the current status of the literature included in Zhi Wang, a total of 484 articles were found. The number of articles per year before 2017 was in single digits, followed by 58 articles in 2017, 158 articles in 2018 and 225 articles in 2019. Thus, in the past three years, scholars have significantly increased their research efforts on the application of artificial intelligence in the field of justice. The research has focused on the following points. First, judicial artificial intelligence, starting from its function and the characteristics of judicial rule and summarizing the many limitations of judicial artificial intelligence, thereby limiting its subject attributes. Second, how judicial artificial intelligence affects the trial mode and the thinking mode of judges, which represents a summary of the technology's application process. The third focus has been judicial artificial intelligence and legal reasoning. Research in this area attempts to construct knowledge maps and logical models that enable machines to learn effectively and bring legal reasoning closer to the computer's digital computing logic. Here, the aim is to investigate judicial referees and establish better practices.

\subsubsection{Practical Application Level}

From a practical perspective, Professor Zhang Lixing of Peking University, set up a search and retrieval system for foreign-related laws and regulations under very tough conditions with his graduate students in 1985. The magic weapon of Peking University that now we are regularly in use is the evolutionary version of it. It is a completely new system for information presentation, capable of legal information data mining and knowledge discovery, and also embraces the function of association between articles in a statute and related cases. A "Practical Criminal Law Expert System" was developed by Professor Zhao Yanguang in 1993. It is composed of reference retrieval system, auxiliary qualitative system and aided measurement of penalty system, with the function of retrieving criminal law knowledge and to make an inferential judgment on criminal cases, in the same year, the "LOA Lawyer Office Automation System" was developed by Professor Hu Zhao. Which takes the function of the case file and legal information management. Professor Zhang (2018) pointed out these research results opened the door to practical research. The developed software has the functions of information retrieval, inference and judgement and has been applied by the judicial department.

In 2016, the Supreme People's Court and the Supreme People's Procuratorate successively proposed plans and offered opinions on the construction of "Zhi Hui Fa yuan" and "Zhi Hui Jian Wu". The Ministry of Public Security, the Ministry of Justice and other departments also issued relevant regulatory documents for informationization. The courts and judicial organs have invested in the construction of "smart courts". In 2016, Hangzhou launched "Fa Xiaotao", which is China's first legal robot (Shen, 2017). The robot has provided clients with information on topics such as lawyers, law firms, courts, and caseloads based on the robot's analysis and analysed and modelled according to judicial data to in- 
vestigate the characteristics and laws of trial execution. Subsequently, Beijing, Shanghai, Suzhou, and other cities launched the "Judge Rui" intelligent research and judgement system, the " 206 " intelligent criminal case assistance system of the Shanghai Court of Justice, the "Intelligent Trial Suzhou Mode" and other intelligent auxiliary software.

The research on judicial artificial intelligence has been targeted and practical. Whether the research object is an information retrieval system or a case- or rule-based expert system, theoretical analyses been developed and such research has been combined with practical investigation.

Early research on the combination of artificial intelligence and the law was primarily led by experts in artificial intelligence-related fields. With the involvement of more experts in the field of law, the research results have assumed more practical value. From an international perspective, it is not only the case-centred Anglo-American-French countries that emphasize the use of artificial intelligence in the judiciary. In addition, traditional civil law countries, such as Germany, France, and Japan, have actively examined relevant issues. Disciplinary integration is closer, and studies have combined Bayesian networks, semantic technology, and ontology. The latest scientific research results have been used in the judicial field to promote the effectiveness of judicial artificial intelligence (Zhang, Yang, \& $\mathrm{Pu}, 2014$ ).

However, in practice, in various places, numerous problems remain. On the one hand, a technical reason is at fault: In the course of reasoning, it is very complicated to accurately describe a case, statutory rules and argumentation, especially to an accurate expression of legal knowledge. Moreover, according to the above knowledge, to make rule-based or precedent reasoning is a running process for the need to understand the mechanism of legal reasoning and the whole trial procedure. The current artificial intelligence system is hard to do, at present. Therefore, at this stage, we need to decompose legal knowledge into individual elements and marking it to enable the machine to identify and understand, forming a complete legal knowledge graph. But the marking workload is huge and the cost can be high. Therefore, the current intelligent software causes problems such as retrieval error, also wrong analysis and decision-making in the process of assistant the judges, resulting in a lower acceptance of them. On the other hand, a design reason is to blame. Professor Zhang (2014) pointed out although current AI-assisted software incorporates the opinions of judges and scholars in the design process, it difficult to acquire judgement experience in specific judicial practice. In terms of the complexity and professionalism of the trial, it is difficult for the machine to arrange the knowledge framework and the knowledge logic. For linking the abstract law with specific case fact requires the systematic and logical thinking of legal experts. The certain terminology, precipitation and accumulation of practical knowledge of case handlers is one of the important sources to refine machine learning content and one of the main tasks that we need to improve in the future. 


\subsection{Principles and Specific Scenarios of the Judicial Application of Artificial Intelligence}

Currently, the intelligent systems established by courts in various locations identify standard request paths based on a standard understanding of language and realize the intelligent analysis of file information. Additionally, such systems can establish a case element database to extract essential elements, index legal norms and implementation cases as well as search facts and legal elements (Ge, 2018).

In addition, the circumstances of extracted legal documents can be structured and mathematical models used to describe the characteristics and rules of the data. Functions can be applied to calculate mathematical models or the algorithms that correspond to them. Artificial intelligence uses simulation algorithms to obtain sentencing references and automate document generation. According to this principle, the intelligent assistant system has many specific application scenarios, such as case push, similar guidelines, prompts, departure warnings and other auxiliary trial intelligence over the entire litigation process. This system is currently the most typical and has application effects. The most well-known example is the Shanghai Intelligent Assistance System. The Shanghai High Court has formulated evidence standards for common crimes and different litigation stages. This is the first time that evidence standards and rules have been embedded in a data-based criminal case handling system of the public security organs, procuratorates, and courts. The Shanghai system can provide guidance in criminal cases, review the single evidence of the case, and judge, check and monitor the evidence chain as well as the entire set of case evidence. Formulating evidence standards, constructing evidence models and other paths as rules for machine learning are sufficient to indicate that the system has begun to focus on the machine study of human legal thinking and adjudication rules to unify evidence standards. These developments as well as constructing evidence models and other paths as rules for machine learning indicate that the application of artificial intelligence in justice has gradually progressed from concept to method.

In short, artificial intelligence has been introduced on the level of filing, trial records, and trials of case entities. From the perspective of the full online litigation process, multi-platform information sharing and docking, algorithm application, and trial guidelines, artificial intelligence has enriched judicial innovation. To judge from the application of technology to document delivery, evidence, and court trials, artificial intelligence has had an impact on the discipline of justice, and the in-depth combination of artificial intelligence technologies, such as artificial intelligence-assisted trials and judicial activities, is related to the comprehensive support of judicial level reform.

However, the application scope of the auxiliary system is narrow. First, from the perspective of the types of cases covered, only the criminal auxiliary case handling system has begun to operate, while the civil and administrative case systems are still being investigated. In addition, the criminal case system only 
covers approximately 10 types of criminal case, in terms of spatial scope, R \& D is limited to local areas, such as Shanghai, Beijing, Guizhou, and Hangzhou. Second, the system has push cases that are inaccurate, excessive, and whose source is unknown, and the actual needs of the judge cannot be addressed. The future direction of efforts should be based on the legal relationship and the focus of the dispute in extracting elements. Third, R \& D efforts and functions continue to require improvement. The use of different $\mathrm{R} \& \mathrm{D}$ companies has resulted in high costs, weak data integration, large practice differences, and shallow R \& D issues.

\subsection{Functional Expectation of Artificial Intelligence Judicial Application}

\subsubsection{Artificial Intelligence Can Improve the "Quality" of Judicial Trials} Judicial AI proponents hope to use the technology to analyse similar cases based on big data, formulate corresponding guidelines for evidence standards, verify and compare such standards, preclude defective evidence, exclude illegal evidence, avoid external interference, and improve judicial credibility. With the help of the Internet, an AI-assisted system can realize the full disclosure of the trial process and the court's case-handling process. As a result, judicial justice can become more transparent. With the help of big data and a unified platform, judicial AI can be used to prompt the case record management system to leave marks throughout the process and achieve the effect of comprehensive supervision of the case trial process. The same type of cases can obtain the same or similar judgement results. Through data sharing, one can achieve the goal of identical cases resulting in the same sentence. In addition, not only can judicial AI provide a new solution for the treatment of difficult cases. It can also facilitate unify the standards of judicial decisions.

\subsubsection{Artificial Intelligence Intervention Can Improve the "Effect" of Judicial Trials}

With the help of speech and image recognition technology and neural network technology, the judge is liberated from repetitive labour. In addition, the time the judge requires to retrieve relevant legal provisions and similar cases is shortened. Moreover, the judge can even realize the one-click creation of referee documents, effectively alleviating the shortage of judge resources. In short, the judicial application of artificial intelligence facilitates litigation procedures, enabling judges to focus more energy on solving difficult problems and improving judicial efficiency.

\section{Analysis of Problems in the Judicial Application of Artificial Intelligence}

Two questions require our attention. The first is whether the ability of artificial intelligence to improve judicial efficiency can be expected to be converted into objective functions. The second is whether the application process complies with 
traditional judicial rule or at least does not violate such rules. In addition, limitations faced in the specific application process and the problems that arise are important practical issues that require our active response.

\subsection{Essential Defect}

General Secretary Xi Jinping noted that the reform of the judicial system should obey the regular pattern of justice, and we must combine judicial system reform with new scientific advances while permitting technology to play a greater role. This view means that we should not focus on applying judicial artificial intelligence to improve litigation efficiency. To reduce litigation and other costs, we should investigate how to integrate judicial work with science and technology to create and apply artificial intelligence products that meet the requirements of judicial reform. To this end, we can examine the judicial application of artificial intelligence and the reform of the judicial system. The purpose of judicial system reform is to improve judicial efficiency and realize judicial justice. Judicial artificial intelligence products can superficially achieve these goals in the application process. However, whether the goal has been really achieved or not is a problem worthy of attention.

Professor Qian (2018) observed that judicial system reform centred on trials proceeds by changing public security organs, procuratorates and the relationship between the courts to avoid the occurrence of unjust and false cases and achieve the goal of justice. However, under the guidance of the unified algorithm, a case is determined to be right or wrong from its beginning, which means that the dominant position of the public security and procuratorial organs cannot be changed. It is a question of changing organizational relationships and structures. Similarly, for the reform of internal judicial relations, judicial artificial intelligence can use data analysis to control the behaviour of judges and strengthen their relationship with the ombudsman and the presiding judge. In addition, the same algorithm has made the relationship between the lower and upper courts more stable. To reform the post system, applied judicial artificial intelligence, not only are judges facing new risks, but also provide ways to avoid them.

\subsection{Prerequisite Defect}

To judge from the operating logic of artificial intelligence, its application cannot be separated from the support of judicial big data and the guidance of algorithms. However, the current status is as follows. First, judicial big data are not comprehensible, reliable, and objective. That is, not all referee documents can be found online, and at least half of them are not online. Even the online referee documents do not contain all the information that can affect the results of the referee decision, pre-trial procedures, and role discussions. The judge's testimony process is generally not public, and even certain judgement documents only record the conclusions of the judges. Judicial data are inaccurate. Certain judgement documents on the Internet represent redundant data, and there are 
even many conflicting judgements and many local practice differences. There are 100 judgements for 100 judges, of which there may be 25 similarities, but similarity does not represent the right direction (He, 2017). Based on the previous data foundation, artificial intelligence will attempt to solve similar problems in the future. However, the same problem may have different solutions in different times. For example, past sentencing was too heavy, and today, the confession and punishment system is applied. The knowledge map for the premise of artificial intelligence's judicial application is incomplete. There are two ways to construct a knowledge map. One is to automatically construct a knowledge graph through deep learning of massive documents. The other is to extract the corresponding plots from the judgement documents, laws and use judicial interpretations, to enrich and form a complete map. Although knowledge graph + deep learning is the mainstream algorithm at the discourse level, in practice, for technical development reasons, the knowledge graph (rather than deep learning) represents the mainstream algorithm (Zuo, 2018). The construction of a knowledge map requires very high levels of data, models, and knowledge granularity, even if the case is a simple one, such as a drunk driving case, and the degree of granularity of the knowledge map is very high. Knowledge map construction requires the case handler to label elements one by one, which is quite time-consuming and labour-intensive. In addition, there are multiple expressions in legal language. For example, the expression of surrender can be a truthful confession or an automatic submission. It is difficult to create a highly accurate knowledge map that can accurately reflect the facts of the case and the judge's reasons for the verdict.

\subsection{Defects in the Process}

The core of the judicial adjudication process is to objectively judge the facts of the case and accurately apply legal rules. First, evidence is the key to restoring the facts of the case. Current judicial artificial intelligence software attempts to simulate the judge's process of evidence reasoning. However, with respect to the judge's ability to judge and support evidence, artificial intelligence is incompetent in terms of force judgement and proof of standard judgement. Second, the true and objective restoration of the facts of the case requires the experience of the case handler, value judgements, and even the spirituality of the judge in certain cases. For example, in divorce proceedings, to make a divorce judgement, the judge must determine that the relationship between the couple has collapsed. In addition to objective evidence, during the trial, the judge can make a comprehensive judgement based on the eyes, language and other behaviour of the couple to determine whether the relationship has collapsed. However, no matter how advanced the technology is, artificial intelligence cannot possess human nature and thus simulate a judge's value judgements. Ji (2018) pointed out that human nature is the origin of feelings and emotions, which is completely different from the intelligence that artificial intelligence can simulate. Judicial artificial 
intelligence software finds it difficult to learn this flexible value judgement of judges. From the perspective of law application, the textual expressions in legal articles are general and abstract. In particular, they lack clear assumptions and express legal facts in legal principles. Such language is also mostly ambiguous, and assistive software may have a biased understanding of the original intentions of the lawmakers (Tu \& Yu, 2018). Different from computer applications in the fields of the board game go and automated driving, judicial artificial intelligence cannot excel on the basis of calculation alone. Current judicial artificial intelligence software finds it difficult to accurately determine the facts of a case and accurately apply legal rules.

\subsection{Outcome Defects}

From the perspective of technological development, the construction of judicial artificial intelligence requires the deep integration and docking of technical personnel and legal experts. Currently, there are technicians who do not understand the court's demands and the developed products are beyond the current level of theoretical legal research. The more straightforward legal theories that technical personnel can understand are transferred to computers. Therefore, it is difficult to make substantial progress in judicial informationization. The construction of artificial intelligence systems requires legal experts to raise theoretical issues, repeatedly discuss them, screen them one by one based on big data and propose solutions. Therefore, in the short term, close cooperation between legal experts and technical experts is required, and in the long term, the training of composite "artificial intelligence + law" talents is on the agenda. To judge from the prospects for the future development of judicial research, such research represents a way to generate and promote legal development rather than only to implement existing laws. The application of judicial artificial intelligence has to an extent undermined the judicial rules and changes in society. Judicial trials must focus on being able to construct new rare cases of rules for referees ("Trial Research" Editorial Board, 2006). In addition, although many cases have similarities in the composition of case elements, making a case judgement requires a logical argument. Even from the perspective of the current application efficiency or accuracy of the software system, the most popular artificial intelligence software lacks an objective logical reasoning ability, and given the opacity of its operation process, the judge and the parties cannot understand the process of the evidence ( $\mathrm{Tu} \&$ Yu, 2018).

\section{Countermeasures for the Application of Artificial Intelligence in the Judicial Field}

According to the principles and specific application scenarios of artificial intelligence products in the judicial field, it is found that the application of artificial intelligence products in the judicial field can improve judicial efficiency. However, our efforts are directed to ensuring that the path and method of its imple- 
mentation are consistent with the traditional pattern. In addition, artificial intelligence faces many limitations in the application process. Generalizations and analogies in legal reasoning, such as in areas that require value judgements and the judge's wisdom, are not suitable for the application of judicial artificial intelligence. Defining and further investigating countermeasure paths will undoubtedly prevent the ill effects of judicial artificial intelligence, thereby indirectly improving judicial efficiency.

\subsection{Clarifying the Auxiliary Status of Judicial Artificial Intelligence}

With the advance of technology, it can be expected that the degree of application of artificial intelligence software to adjudication will increase. Therefore, it is urgent to clarify the attributes of auxiliary tools for artificial intelligence software. Such software has become an inevitable trend in the field of adjudication. Under the circumstances, how to address the relationship between assistive software and judges is the most important issue we face. The relationship between intelligent assistive software and judges is reflected in the following two aspects. One is different from the previous scientific and technological revolution represented by artificial intelligence. The software has the ability to analyse and judge. Therefore, it is not a one-way tool for the judge. It can thoroughly study legal documents, such as previous judgements, and make decisions on the current case. This ability will challenge the dominant position of the judge.

Second, the conflict between 1) the rationality generated by the analysis and decision-making intelligence of artificial intelligence software and 2) the traditional rationality of judges has led us to reconsider what the rationality of judicial decisions should be. This reconsideration is likely to change certain of our previously held views (Chen, 2018). At this stage, we cannot determine the thinking logic and operation mode of artificial intelligence software-assisted trials, let alone the extent to which they will resemble the traditional judge's thinking mode. Therefore, it is particularly important to clarify the auxiliary status of intelligent software. The core of this question is the division of responsibilities. We can imagine how to divide responsibilities between software designers, judges, and intelligent software during trials. This requires reviewing trial responsibilities. Trial responsibilities include factual defects, evidence defects, legal flaws, clerical flaws, and other errors. Therefore, we will face a large number of perplexing situations, such as what to do when the machine provides wrong information leading to trial errors and when it is difficult to review the rationality of the algorithm. For example, what if the judge adopts information provided by the intelligent software but later discovers the information is wrong. Can this mistake be viewed as the software's responsibility, thereby diminishing the responsibility of the judge? If the intelligent software can be considered to bear the responsibility, does this mean that the intelligent software has the status of a trial subject or can have a legal personality? These and other issues must be clarified.

Obviously, as in other industries, the emergence of artificial intelligence has 
brought about the "replacement" of human perspectives, and scholars have responded to this concern in their articles. Professor Ji (2018) noted that scientific and technological means are only auxiliary means in judicial practice. Do not "place the cart before the horse"; otherwise, the judicial power will be led astray. $\mathrm{Wu}$ (2017) explains from the "cognitive" defect level of the computer that although artificial intelligence technology is meaningful for the study of legal issues it cannot realize the intelligentization of judicial trials. This inability actually illustrates the irreplaceability of legal professionals in legal practice. Guo (2017) uses literary works to explain the primary and secondary relationship between humans and artificial intelligence in legal practice. The judge observed that the first priority of judicial artificial intelligence is to assist and serve the judge in handling a case. In the final analysis, the task of the court's artificial intelligence is to integrate the judge's wisdom and make good use of it (He, 2017). One should define artificial intelligence with clear legal norms. It is advisable to combine the software with human judges in its trial support status and to use procedural safeguards to regulate the specific circumstances of its auxiliary judgements to prevent the risk of trial errors.

\subsection{Defining the Application Scope of Intelligent Software}

In terms of application, even if we insist that the goal of applying artificial intelligence in the judicial field is to assist judges in handling cases, we must formulate systematic and forward-looking basic rules. It is necessary to establish a corresponding roadmap and basic framework for intelligent judicial reform to clarify the scope of application. At the present stage, if legal norms are to be formulated to clarify judicial AI's scope of application, the formulated legislative norms can only be in principle, not operational, and it will be difficult to establish regulatory effects. The theoretical and speculative methods being actively discussed in the academic world to analyse the differences between artificial intelligence and human beings will be ineffective and not conducive to preventive measures. The preferred measures are as follows. First, one must predict the general development stage of artificial intelligence in the judicial field. We are required to formulate the relativity required by judicial artificial intelligence in different stages of development (i.e., reasonable differences in the application of substantive laws in different regions), reliability (to prevent useless and harmful data), and appropriateness (the application of artificial intelligence systems must not be subject to transitional enforcement standards because judicial judgements are discretionary judgements in addition to being prescriptive judgements). (A reasonable space for discretion is required.) Independence is another issue. (i.e., how do public security organs, supervisory organs, and judicial organs handle cases in an intelligent system to ensure that the principle of exclusive investigation, prosecutorial, and judicial power is implemented? Is it possible to guarantee a normal relationship between powers? Therefore, it is necessary to clarify the research and development basis, application impact, and implementation 
plan of intelligent systems). A further concern is controllability (i.e., data control rights: cases that should ensure data related to generation rules, experience accumulation, and skills transfer must be handled independently by judicial officers) and other requirements. AI's application in the judicial field should be clearly delimited. Informal systems and unstable case rules are restricted areas of application. Third, the specific application of artificial intelligence systems, such as the case push function, requires attention. For example, currently, there are no normative documents that specify similar time guidelines (e.g., can judges refer to cases in recent years?), space guidelines (i.e., whether local courts should prioritize decisions by higher courts), validity issues (e.g., if there is a conflict, is it always subject to the decision of the High Court? Who controls the standards?). We must also clarify the conditions, standards, nature, type and scope of the application of assistive software and explain the reasons for its application. That is, a set of national standards, standard processes and management mechanisms are required.

\subsection{Implementing a Differentiated Trial System and an Ex Post Supervision System}

Drawing on the simplified procedural system of civil lawsuits and the expedited procedures of criminal lawsuits, we will formulate a differentiated trial system to support the application of artificial intelligence in simple cases and conduct more refined and precise treatment of its core factors, saving more resources to be used in complex cases. However, differentiated trials do not mean a complete separation between the two but a benign interaction. The main role and side effects in different cases are replaced with one another, forming a novel judicial trial mode. The transparency of justice conflicts with the privacy of algorithms. It is difficult to control or explain the problems generated by artificial intelligence. Therefore, the method of ex post monitoring is more desirable. The constitutive elements currently envisioned include but are not limited to the subject of supervision, the object of supervision, the specific form of supervision, and other general procedural issues. The core is the court's reasonable interpretation or amendment of the judgement made by artificial intelligence, which can not only balance the interests of all participants in the lawsuit but also effectively improve the acceptance of the verdict.

\subsection{Formulating Ethical Norms for Judicial Artificial Intelligence}

It is necessary to implement ethical design concepts, embed human norms and moral values into the system, formulate ethical guidelines for users and designers of intelligent assistance systems, and implement them in judicial practice. Soft law is used to make up for the lack of flexibility of hard law. Specific measures include but are not limited to the following points. The algorithm can be disclosed to a limited extent, at least the review agency. The person in charge of the verification is obliged to observe confidentiality and is at least required them 
to disclose the technical parameters of the system and the degree of impact on sentencing work, such as sentencing. The court should have the right to force the disclosure of the code of the relevant intelligent analysis tool algorithm. When suspicious consequences occur, the designer is required to explain the design principle of the algorithm, and if there is a fault, it should be investigated. Regarding responsible entities, at this stage, it is preferable to establish an ethics committee or artificial intelligence court that combines computer-related experts and legal experts to implement the application of relevant rules specifically for the purpose of risk prevention rather than dispute resolution (Zheng, 2017). Can we learn from the judge warning system developed by the State of Wisconsin, which fully reminds the public of the risks of the judge system?

The specific regulatory paths that can be selected include establishing a moral system, formulating ethical rules for artificial intelligence design, establishing top-down regulatory paths (such as utilitarian artificial intelligence subjects), using deep learning capabilities of artificial intelligence, and configuring modules in artificial intelligence systems. There is also a bottom-up regulatory path. A top-down and bottom-up combined regulatory path is proposed based on the evaluation of the two paths described above. For example, the top-down regulatory path can guarantee an artificial intelligence system is constructed according to a general guiding principle. However, its disadvantages are manifested in the inability of humans to seek a unified standard. The bottom-up regulatory path helps integrate basic theories in different stages and different environments. The integration is very good, and the width of choice will also expand with respect to the challenges facing systems with certain moral capabilities. Therefore, a regulatory path that combines top-down and bottom-up approaches is an important way to solve the ethical problems of artificial intelligence.

\section{Conclusion}

The application and development of artificial intelligence technology in the judicial field are very fast. It is predictable that the intelligence technology will be used more and more widely in cases where the facts are fully proved and easier to type, even in difficult and complicated cases, the new trial mode of "human-computer collaboration" may be realized. It is mainly based on the neural network and machine depth learning theory breakthrough. Improving the application efficiency of technology in the judicial trial field is the direction of our future efforts. The contribution of this article is that it tries to explain the entire process of the judicial application of artificial intelligence based on close integration into the traditional judicial pattern. This study pays close attention to the development of existing theoretical research and tries to choose the optimal operating framework. Starting from the theory of judicial artificial intelligence and the regular judicial pattern of Chinese litigation procedure's principle and proof mechanism, the paper analyses the theory and practice of artificial intelligence applied in the judicial field based on the internal and external factors affecting 
judicial decisions. The paper seeks to demonstrate the function, functional expectations, limitations and risks of artificial intelligence in the litigation field. In addition, it analyses the legal aspects of artificial intelligence, the ethical issues generated by the development of artificial intelligence, the response stimulus, the thorough integration bottlenecks of artificial intelligence and legal reasoning, and possible solutions to resolve problems. This paper is mainly about an overview of phenomenon interpretation and general rules, that is, the judicial application process of artificial intelligence is discussed from the macro perspective. However, in order to realize the successful operation of artificial intelligence in the judicial field, the application of artificial intelligence technology in the judicial field needs to be studied from the macro-level as well as the micro-level. Therefore, the deficiency of this article lies in the lack of necessary thinking on how to ensure the implementation and carry out the concept of fair trial in the process of application when it faces very detailed and important problems, such as how to achieve the balance between "fairness" and "efficiency" in the process of application.

\section{Conflicts of Interest}

The author declares no conflicts of interest regarding the publication of this paper.

\section{References}

Chen, J. H. (2018). The Legal Challenges of Artificial Intelligence: Where Is the Start. Journal of Comparative Law, No. 5, 136-148.

Guo, F. M. (2017). Correct Judgment of the Judicial Position of "Smart Court". https://www.chinacourt.org/article/detail/2017/08/id/2953971.shtml

Ge, X. (2018). The Reality and Prospects of the Application of Artificial Intelligence in Judicial Practice-Based on the Intelligent Aided Case Management System of Shanghai Court Administrative Cases. ECUPL Journal, No. 5, 67-76.

He, F. (2017). How Far Are We from "Judge Alpha"? Fangyuan Magazine, No. 5, 58-61.

Ji, W. D. (2018). Changes in Judicial Power in the Age of Artificial Intelligence. Oriental Law, No. 1, 125-133.

Qian, D. J. (2018). The Chinese Process of Judicial Artificial Intelligence: Functional Replacement and Structural Strengthening. Law Review, No. 5, 138-152.

Shen, Y. F. (2017). When the Law Meets Artificial Intelligence. Fangyuan Magazine, 14, 22-27.

Tu, Y. Q., \& Yu, H. (2018). Interventional Evolution of Artificial Intelligence in Judicial Trials. Journal of Southwest University of Political Science and Law, No. 3, 48-55.

“Trial Research" Editorial Board (2006). Trial Research 2005 (13th Series). Beijing: Law Press.

Wu, X. Y. (2017). The Possibility and Limits of Modeling Judicial Decision-Making in Artificial Intelligence. Zhejiang Social Sciences, No. 4, 51-57.

Zheng, G. (2017). Artificial Intelligence and the Future of Law. Exploration and Free Views, No. 3, 78-84.

Zhao, G. F. (2017). From the Perspective of Practice, the Paper Discusses the Problems 
and Countermeasures in the Construction of Smart Court. https://www.chinacourt.org/article/detail/2017/11/id/3085397.shtml

Zhang, B. S. (2001). Jurisprudential Thinking of Artificial Intelligence Legal System. Law Review, No. 5, 11-21.

Zhang, B. S. (2018). Artificial Intelligence Legal System: Two Difficulties and One Paradox. Journal of Shanghai Normal University (Philosophy and Social Sciences Edition), No. 6, 25-41.

Zhang, N., Yang, S. Q., \& Pu, Y. F. (2014). A Review of Advances in Artificial Intelligence and Legal Studies Abroad. Legal Method, No. 2, 458-480.

Zhang, N. (2014). An Analysis of China's Judicial Discretion Model-A Case Study of Medical Spiritual Damage Compensation. Legal Method, No. 1, 280-298.

Zuo, W. M. (2018). Some Thoughts on the Application Prospects of Legal Artificial Intelligence in China. Tsinghua University Law Journal, No. 2, 108-124. 